use in low doses is increasing, possibly due to its sedative qualities, tolerability, low risk of extrapyramidal symptoms and to limit the unnecessary use of benzodiazepines. However, previous research highlights the risk of metabolic consequences even in low doses. Our aim is to establish the prescribing patterns and off-label use of quetiapine within a complete comminity mental health team population (CMHT).

Method. The GR1 CMHT provides care to a population of 25,000 people in a mixed urban and rural area. Multi-disciplinary case notes for all registered patients were reviewed for a one-year period. A database was created to include sociodemographic details, diagnosis, and medication. The proportion of patients prescribed quetiapine was identified and the dosage divided into multiple increments. The team's consultant reviewed and verified all ICD-10 diagnoses. Quetiapine dose by diagnosis was examined using descriptive statistics.

Result. Of 246 registered patients, 62 (25\% of CMHT caseload) were prescribed Quetiapine. Quetiapine was prescribed across a range of disorders including psychotic 17 (27\%), mood 18 (29\%), anxiety $14(22 \%)$, personality disorders $11(18 \%)$ and others 2 (3\%). Doses spanned between $25 \mathrm{mg}-800 \mathrm{mg}$ daily. 19 patients $(31 \%)$ were prescribed less than $25 \mathrm{mg}, 20$ patients (32\%) between $25 \mathrm{mg}$ and $100 \mathrm{mg}$ and 23 patients (37\%) above $100 \mathrm{mg}$. In psychotic and mood disorders, dosage varied widely between the low and high range. Furthermore, of the psychotic disorders, $11(65 \%)$ were prescribed a second antipsychotic medication. For diagnoses in which the prescribing indication was clearly off-label, the dosages were predominantly low $(100 \mathrm{mg}$ or less).

Conclusion. Quetiapine was commonly prescribed in our patient population. Its frequent off-label use in low doses suggests that its prescription was for its additional qualities. Our findings highlight the importance of assessing the risk-benefit profile for every patient given the potential side effects, involving patients in the consultation of its off-label use and appropriate monitoring.

\section{Audit of compliance with WHO surgical safety checklist (modified for electroconvulsive therapy including NPSA advice)}

Faisal Alam*, Rizwan Ashraf, Kyaw Sein and Terri Feeney

GMMH NHS Foundation Trust

${ }^{*}$ Corresponding author.

doi: 10.1192/bjo.2021.213

Aims. This audit aims to evaluate the compliance with the WHO surgical safety checklist during the electroconvulsive therapy treatment in ECT clinic at Greater Manchester Mental Health Bolton Directorate. The audit is based on WHO surgical safety checklist modified for ECT including National Patient Safety Agency advice. The goal is to improve the compliance and in turn improve clinical outcomes.

Background. The WHO surgical safety checklist (modified for Electroconvulsive therapy including NPSA advice) is devised to promote patient safety, improve teamwork, reduce errors/adverse events and improve overall quality of care. An audit was completed regarding the compliance with the safety checklist at the Bolton ECT clinic and to assess how this could be improved.

Method. Following approval from the clinical audit department, GMMH NHS Foundation Trust, 20 checklists from randomly selected patient ECT files were included in this audit. We looked at whether the checklists were completed, signed and dated. Our current WHO surgical safety checklist is as per the Electroconvulsive therapy accreditation service standards.
Result. A total of 20 WHO surgical safety checklists were reviewed. 95\% of the checklists (19/20) were completed by the duty Psychiatrist. 1 form was not completed. 25\% (5/20) were not signed rendering them invalid. A total of $75 \%$ checklists were complete and valid. Checklists were present in all the case notes.

Conclusion. Compliance with the WHO surgical safety checklist during the electroconvulsive therapy treatment can be challenging due to various reasons ranging from time pressure to difficult clinical situation. This audit has highlighted that the overall compliance with the set standards $(100 \%$ completion $)$ was not achieved. A repeat audit will be important to further improve the compliance and overall clinical outcome.

An audit addressing the quality of prescribing sodium valproate in early intervention service

Humaira Aziz ${ }^{1 \star}$, Khushboo Khatri ${ }^{2}$ and Sneha Upadhyaya ${ }^{2}$

${ }^{1}$ Birmingham Community Healthcare NHS Foundation Trust and

${ }^{2}$ University hospital Birmingham

${ }^{\star}$ Corresponding author.

doi: 10.1192/bjo.2021.214

Aims. This Audit aims to review prescribing practice concerning Valproate in early intervention services.

Method. The audit was undertaken across four EI hubs in Birmingham. Audit standards were derived from POMH-UK (Prescribing Observatory for Mental Health) QIP. Drug cards of the entire EIS caseload in November 2020 were reviewed to identify patients on any preparation of Valproate. A total of 31 patients were identified. Electronic notes of all the patients on Valproate were reviewed to compare prescribing practices against national standards.

Result. A total of 31 patients were prescribed sodium Valproate. All these patients had target symptoms documented in their notes. Reason for starting Valproate was mostly documented as agitation and aggression rather than elation in the mood. In was unclear if patients had full physical health checked before starting Valprotae as in majority (94\%) valproate was commenced as an inpatient. Not all cases had detailed inpatient discharge notes making it difficult to fully understand the rationale for starting Valproate.

$55 \%$ of the patients were on an off-license valproate preparation. Where used off-license majority (93\%)of these patients had no documentation of the rationale behind off-license use. Similarly, in most cases (93\%)there was no evidence of off-license use being discussed with the patients. Most patients had received adequate monitoring in the community (74\%) although there was limited documentation of screening for common side effects. Prescribers were noted to be using Semi-sodium Valproate and Sodium Valproate interchangeably despite these not being bioequivalent.

Conclusion. We recommend that

1. Periodic treatment reviews should document the assessment of response and screening for side effects.

2. Where used clinician should clearly discuss and document the off-license use with patients. $500 \mathrm{mg}$ Semi-sodium valproate (Depakote) is approximately equivalent to $433 \mathrm{mg}$ Sodium Valproate (Epilim). If switching from Semi-sodium Valproate to Sodium Valproate, a slightly higher (approximately 10\%) dose of Sodium Valproate should be used.

3. Unless clear evidence of affective illness is identified, the ongoing need for Valproate should be regularly reviewed by the clinicians. 
Compliance with Driver and Vehicle Licensing Agency guidance in a psychiatric inpatient setting

Nishanth Babu-Mathew ${ }^{\star}$, Sasha Chard and Sarah Winfield

Merseycare NHS Foundation Trust

${ }^{*}$ Corresponding author.

doi: 10.1192/bjo.2021.215

Aims. Driving is complex, requiring adequate: attention and concentration, memory, insight and understanding, judgement, planning and the ability to self-monitor1. Psychiatric illness, and associated medications, may affect patients' ability to drive safely. The DVLA is responsible for determining individuals' safety to drive and produces guidance specific to psychiatric disorders. Patients must comply with relevant guidance and clinicians must determine patients' driving status and offer appropriate advice about medications and any need to inform the DVLA. This audit aimed to determine the compliance with DVLA guidance on a single inpatient psychiatric ward within Merseycare NHS Foundation Trust, UK.

Method. A retrospective review of electronic patient records was completed. Clerical staff identified all patients admitted to Windsor House from 1/8/20-30/11/20 $(n=42)$. Data relating to driving status and driving advice were collected onto individual patient audit proformas, and uploaded to the online Audit Management and Tracking (AMaT) system.

Result. $100 \%$ of patients had diagnoses that would require the DVLA to be informed and $100 \%$ were prescribed medication with potential side effects that could impair ones' ability to drive safely such as dizziness, drowsiness or impaired concentration2. Driving status was only documented for 12 patients (29\%) and type of vehicle driven for only 6 patients ( 1 of whom had an HGV licence).

Discussion of DVLA guidance within the last $3 / 12$ by the mental health team was documented in $17 \%$ patients. Of these patients, appropriate driving advice was given to $86 \%$. All patients advised to cease driving were willing to. No patients were advised about side effects of medications on driving. No notes evidenced if the DVLA had been informed of patients' admission, diagnosis or medication regimes.

Conclusion. Discussing diving status and DVLA advice with psychiatric patients is important but may not always happen in inpatient settings, despite most patients having a relevant diagnosis. Failure to determine driving status may mean some patients are not being given appropriate guidance as required. Counselling on medication side effects in relation to driving should be encouraged as the majority of patients are taking prescribed medication that can potentially impair driving. Recommendations to improve compliance include: adding "driving status" to admission clerking and ward review proformas, educating staff to actively discuss driving with inpatients and create discharge checklists which prompt discussing driving status, medications and driving advice, and to re-audit in 6 months time.

\section{Audit on prolactin monitoring for patients on oral} risperidone, intramuscular risperidone, and intramuscular paliperidone

\section{Mohamed Bader}

Aneurin Bevan University Healthboard

doi: 10.1192/bjo.2021.216

Aims. The aim of this audit was to investigate whether sufficient Prolactin monitoring was completed in a patient sample in the
Torfaen area of Aneurin Bevan University Health Board. This audit targetted patients an oral or intra-muscular formulation of Risperidone in the year 2018 with the hypothesis that Prolactin monitoring is done less frequently than recommended.

Background. Risperidone is the anti-psychotic drug most frequently associated with hyperprolactinemia which is often asymptomatic but can present with symptoms of oligomenorrhea, amenorrhea, galactorrhea, decreased libido, infertility, and decreased bone mass in women. Men with hyperprolactinemia may present with erectile dysfunction, decreased libido, infertility, gynecomastia, decreased bone mass, and rarely galactorrhea. The BNF advises monitoring of Prolactin at baseline, after 6 months, and then annually.

Method. Retrospective review of 150 patients' clinical letters to identify if they are on the above medications, using the local digital records system EPEX. Emails were also sent to community psychiatric nurses asking them if they could highlight any patients they were caseholding on the above medication. Depot clinic lists were also examined. Patients identified as being on the above medication had their blood tests reviewed on the online system Clinical Workstation (CWS) to determine whether they had their Prolactin level tested. A single spot sample of all patients on Talygarn ward in January 2019 was also included.

Result. 1. 28 Risperidone

2. 23 of 28 never had any Prolactin measurements

3. 2 of 28 patients had the appropriate level of monitoring done for the year of 2018

a. One patient complained of Galacotorrhea

b. Another patient had baseline done while on the ward and isn't due for any further monitoring at the time of writing.

Conclusion. The above results identify that Prolactin monitoring is not being routinely completed for patients on the studied medication at an acceptable compliance level. Limitations around utitlity of prolactin monitoring may be the contributing factors; eg. Prolactin levels or medication dose may not be positively associated with adverse effects.. Further efforts were made to highlight the importance of baseline prolactin monitoring, as well as including a baseline Prolactin as an admission blood test for patients presenting with psychotic symptoms or on an antipsychotic. A complete audit of metabolic monitoring and Prolactin levels for all patients on anti-psychotics would be an appropriate next step.

The hidden cost of not having a dual diagnosis team (an audit looking at inpatient admissions for Redbridge Community Recovery Team West)

Madeeha Bandukda*, Muhammad Aadil Bhenick, Najam Chaudry and Henok Getachew

NELFT

${ }^{\star}$ Corresponding author.

doi: 10.1192/bjo.2021.217

Aims. Co-existing mental illness and substance misuse is highly prevalent within the UK, with approximately $40 \%$ of people diagnosed with psychosis having a history of substance misuse. However, in Redbridge we currently do not have access to a dual diagnosis team or integrated care.

This audit aims to assess the health and social implications of fragmented care, plus the effectiveness of mental health services in assessing patients with dual diagnosis and referring to specialist misuse teams. We used the NICE guidelines on co-existing severe mental illness and substance misuse [CG120] to help guide our recommendations. 